\title{
Lumbar puncture: low-cost interventions improve efficiency and patient experience
}

\author{
Authors: Alison Berner, ${ }^{1}$ Claudia Craven, ${ }^{2}$ Faidra Laskou, ${ }^{1}$ Neil Graham ${ }^{2}$ and Gordon Ingle ${ }^{2}$
}

\section{Aims}

To improve the experience of patients undergoing lumbar puncture (LP) by standardising the process of consent and by improving ease of equipment preparation.

\section{Methods}

We conducted an initial survey of core medical and neurology trainee doctors on consent practice in a specialist neurology hospital. Over a 2-week period, data on LPs carried out on the wards were prospectively collected. Patients rated their understanding of LP and experience of the consenting process using a visual analogue scale, where zero was 'totally disagree' and 10 was 'totally agree'. Time taken to prepare equipment was recorded.

An information leaflet was designed to assist in explaining the procedure to patients. Doctors were educated on best practice for gaining consent, with incorporation of instructions into the local junior doctor handbook. Stickers were designed to assist documentation of risks discussed. Regularly stocked LP trolleys containing equipment necessitated for best practice were introduced onto the wards.

Patient satisfaction and equipment collection time were resurveyed over 2 weeks.

\section{Results}

Average time to collect equipment improved dramatically from 13 minutes at baseline $(n=14)$ to 5 minutes following the introduction of dedicated trolleys $(n=9)$.

Prior to introduction of stickers and guidance for trainees, $47 \%$ of doctors $(n=17)$ documented consent in the notes, with only $30 \%$ using a trust consent form. $18 \%$ provided supplementary materials, with considerable variation in the numbers of doctors discussing each of the frequently occurring or rare yet serious risks.

At baseline, patients rated their understanding of the reason for the procedure, the process and risks involved at 8.8/10 $(\mathrm{n}=10)$. Average score for recalling the information provided was $8.8 / 10$ and for being able to explain to another person

Authors: ${ }^{1}$ University College London Hospitals, London, UK; ${ }^{2}$ National Hospital for Neurology and Neurosurgery, London, UK was 6.9/10. Following interventions $(n=9)$, average score for understanding the reason for the procedure rose slightly to 9.1, while understanding of risks and what LP involved were unchanged. Recall of information given score dropped slightly to $8.4 / 10$, while ability to re-explain rose to $8.5 / 10$.

\section{Conclusions}

Implementation of dedicated LP trolleys dramatically improves efficiency in this common procedure. The process of gaining consent was found to be highly variable: we demonstrated that this can be standardised simply and effectively with leaflets and stickers to assist documentation while modestly improving aspects of patient understanding. There is further potential for improvement and we hope to achieve this in due course by incorporating other media such as video. 